Indonesian Journal of Electrical Engineering and Computer Science

Vol. 11, No. 3, September 2018, pp. 1035 1041

ISSN: 2502-4752, DOI: 10.11591/ijeecs.v11.i3.pp1035-1041

\title{
Study of Load Optimization and Performance Issues in Cloud
}

\author{
Madhina Banu D, Aranganathan \\ B.S.A Crescent Institute of Science and Technology, Chennai, Tamilnadu, India
}

\begin{tabular}{|c|c|}
\hline Article Info & ABSTRACT \\
\hline $\begin{array}{l}\text { Article history: } \\
\text { Received Feb 9, } 2018 \\
\text { Revised Jun 2, } 2018 \\
\text { Accepted Jun 11, } 2018\end{array}$ & $\begin{array}{l}\text { Nowadays, cloud computing is the latest computing platform which is } \\
\text { feasible to the user for computation and unlimited storage and data } \\
\text { transmission with minimal cost and time in a cloud environment during the } \\
\text { internet. The load balancing is important criteria of cloud environment that } \\
\text { avoid same nodes overloaded and others are idle. Ultimately load balancing } \\
\text { can enhance the QoS parameters including make span, cost and resource }\end{array}$ \\
\hline $\begin{array}{l}\text { Keywords: } \\
\text { Cloud computing } \\
\text { Quality of Service } \\
\text { Cloud performance } \\
\text { optimization } \\
\text { Load balancing optimization } \\
\text { Scheduling } \\
\text { Virtualization } \\
\text { Data center executions }\end{array}$ & $\begin{array}{l}\text { properly utilized federation mechanisms, which offers physical resources } \\
\text { based on demand to maintain the cloud application efficiency. However, the } \\
\text { technique failed to optimize load where part of the servers suffering from } \\
\text { heavy load after an execution of the application. In the current scenario, } \\
\text { several systems are facing same kinds of problem which is the biggest cause } \\
\text { to increase the virtual machine (VM) cost. Current systems still have a time } \\
\text { delay, request-response process error from data center side in cloud } \\
\text { environments. To overcome these issues, the research study studies all } \\
\text { related technique for cloud performance optimization and load balancing } \\
\text { issues. The main framework objective is to offer an effective solution to } \\
\text { store/search/transmit/data with minimal cost and time without compromising } \\
\text { the QoS constraints. The study represents different policies and cloud- } \\
\text { specific strategies to enhance the performance of cloud application with } \\
\text { minimal cost and time. The research study is also planning to find out an } \\
\text { effective solution for traffic, data congestion and media streaming issues in a } \\
\text { cloud environment. }\end{array}$ \\
\hline
\end{tabular}

Copyright (C) 2018 Institute of Advanced Engineering and Science. All rights reserved.

Corresponding Author:

Madhina Banu D,

PG \& Research Department of Computer Science,

Presidency College, Madras University,

Chennai-60005, Tamilnadu.

Email: madhinamca05@gmail.com

\section{INTRODUCTION}

Virtualization technology has been introduced and minimizing the physical assets utilization with nominal cost and high demand. Cloud Computing contributes the services in three types at different stages such as infrastructure as a service (IAAS), platform as a service (PAAS), and software as a service (SAAS). Particularly, IaaS provides clients the physical assets in the structure of the VM to execute cloud service provider in the data center whereas PaaS and SaaS serve for exact key stacks and application like suites of software. The cloud computing utilizes a large group of servers with low-cost PC technology with specialized connections to spread data-operation. The computing prefers distributed IT infrastructure for interconnecting the large pool of users. The technique automatically controls and optimizes resource by using leveraging a metering capability according to the type of services like processing, storage space, and bandwidth utilization and profile of client. Resource utilization monitored, managed, frequently updated to cloud service provider and as well users.

To optimize the load, the existing system developed federation mechanisms, which offers physical resources based on demand to maintain the application efficiency. However, the technique failed to optimize 
load balancing where part of the servers suffering from heavy load after an execution of the application. The response time on the servers took long waiting time to process the process the service due to the unnecessary response time delay, which is unfavorable for real-time web-based applications. In another way, distributed computing environment has the complexity of load optimization during the request-response process. The job arrival pattern is unable to predict the load on every data center each node for load balancing. The system is still suffering from time delay, request and response time is sues from data center is sues. Cloud infrastructures are required to predict the cost-benefit for equivalent Quality of Service (QoS) by qualified clients. However, simulation is not compatible for statically investigation, due to a large number of simulation parameters.

In [1] introduced fuzzy logic based energy-aware autonomic as set scheduling strategy and an energy was well-organized training in data centers. It mapped cloud workload to improve resource utilization and energy consumption. In [2] illustrated adaptive virtual machine asset scheduling methodology depends on auction strategy consider various features together with system bandwidth and auction target. Method sequences, the clients' bids provided the competition target with minimum costs. In [3] described a robust routing method which is arrived at higher network energy effectiveness. In [4] identified a DVFS-enabled Energy-proficient Workflow project Scheduling strategy which is leveraged useful slot time during severs migrations. The technique estimates primary scheduling order of all jobs and acquired the complete makespan \& target. In [5] designed a scheduling algorithm for the task to enhance the worth of service and it overcomes a starvation of low priority queues (extended waiting precedence queue). It offers fairness at main concern level by developing an integration of the main concern queue and round-robin methodology.

In [6] explained dynamic energy-proficient VM migration and consolidation methodology which was depends on a multi-resource energy proficient method. The technique minimized energy utilization through QoS guarantee. In [7] deigned a finite multi-server queuing model with queue dependent heterogeneous servers reducing the waiting time. In [8] reviewed cloud computing simulators to appraise the performance of load balancing in the cloud environment. In [9] designed resource allocation mechanism for machines, which depends on the morality of coalition configuration and the hesitation of game theory. In [10] introduced Combinatorial Double Auction Resource Allocation (CDARA) market strategy to evaluate the effectiveness from an economic perspective. It permits competition and bidding on both sides for unlimited amount of products.

In [11] studied about mobile cloud computing including the definition, architecture, and applications to fulfill the user requirement. In [12] explained an Inter-Cloud Mediation Service to engage open clouds formation a two-way environment for allocation and general as sociation of cloud computing services. In [13] expressed truthful online cloud auctions framework to assure truthness with heterogeneous and online user demand. In [14] introduced an Aneka tool which is a venture Cloud computing resolution. It harnesses the authority computational assets in relying on confidential and open Clouds and conveys it to clients. In the tool tackled diverse scenarios: from business applications to computational science. In [15] addressed a Generalized Priority strategy for proficient task implementation with FCFS and Round Robin Scheduling techniques. It manages the availability of CPU memory and scheduling policy.

In [16] reviewed and analyzed the most practical network issues to the provision of high-assurance cloud services through the Internet. In [17] developed a Service Composition Optimal-Selection problem was solved by complete as sociation based similar adaptive chaos Optimization with reflex mig ration (FC-PACORM) algorithm. In [18] explained Green Scheduling methodology which integrate neural network interpreter for optimizing server power utilization in Cloud computing environment. The system turns off unutilized cloud servers and restarts to diminish the amount of running cloud servers. In [19] expressed multi-objective genetic strategy (MO-GS) and it reduced energy expenditure and developed the revenue of cloud services under the restriction of aspects. The method contains many components to a nalyze the cloud application, and allocate the suitable resources. In [20] explained Deadline Aware Two Stage Scheduling algorithm and it allocated Virtual Machines (VM) for the requested operation which was gotten from the clients. The method allots VM's as assets to the requested tasks based on the time taken to process and disperse tasks by considering time limits.

In [21] introduced task-oriented resource distribution model by using pair wise association matrix procedure and the Analytic Hierarchy Process depends on the resources available \& preferences given by clients. In the technique allocates resources depends on the ranking of operations. In [22] elaborated a Discrete Symbiotic Organism Search (DSOS) methodology which optimized task allocates on cloud computing assets. The technique utilized to resolve numerical optimization issues. In [23] developed a communication-aware method of cloud Applications (CAMCA) to calculate the vertices together with separate vertices to signify announcements. The method generates opening for optimization of existing results to improve the efficiency of resource allocation. In [24] used virtualization mechanism for dynamic allocation of data center as sets depends on the application requires and optimization of cloud servers, Green Computing is facilitated. In the technique introduces the idea of "skewness" to estimate the inequality in the 
multi-dimensional asset expenditure. In [25] expressed EMUSIM and it extracted data from application behavior through emulation automatically. The simulator utilized the data to produce consequent simulation method.

In [26] depicted the purpose of the cloud paradigm which enhances the use of network that provided the capabilities of utilizing one node from another node. It described the load balancing between the clients and the servers. The load balancing was highly utilized in service provider resources. It also helped the resources to be utilized at large in data centers which make the performance of the system higher leading to client satisfaction. In [27] highlighted the tradeoff for offloading. The work provided architecture. The genetic algorithm integrated mobile cloud computing for the purpose of automatic offloading in enhancing the system response time. Also energy consumption minimized for mobile devices. It developed optimal offloading algorithm for the mobile devices of user with cloud availability and load of user's mobile devices. The method provided the solution and formulation of the Markov Decision Process, MDP model for minimizing the offloading cost and computational cost. In [28] explained load balancing plays vital role in cloud performance and its stability. It discussed various load balancing algorithms which helped in distributing the load among the nodes and founded which suited the most.

To overcome these issues, proposed research plan studies all related technique for performance optimization and load balancing scheduling is sues. The study also focuses on traffic issues and data center processing delay in cloud environments. The research work main motto is to fulfil the gap between current requirement and available technologies. The research work represents the different policies and cloudspecific strategies to enhance the performance of cloud application with minimal cost and time. The method can also reduce the bandwidth issues \& minimizes the data center response time delay. The technique minimizes the energy consumption for improving the QoS(quality of service). In proposed load balancing scheme, load balancer restrains the track of some cloudlets currently being processed by every VM and attempts to maintain the active load. The model has a main controller, and it balances load and analyzes the information. The system offers balancing of load predictions depending on the preference of load balancing strategy. In the paper contributions are given below in details:

1. To study all interrelated technique for presentation optimization and load balancing scheduling issues.

2. To present different policies and cloud-specific strategies to get better performance of the cloud application at least cost and time without compromising the QoS constraints.

3. To find out an effective solution for traffic, data congestion and media streaming issues in a cloud environment.

The rest of papers of structures are followed as Section 2 address the related work which elaborates the various technology functionality and feature details which are closest to proposed research work plan. Section 3 explains the current approaches and similar study details to understand the current needs. Section 4 concludes overall review work with future research work plan.

\section{RESEARCH METHOD}

In the section explains the available approaches with their feature and functionality details related to cloud performance optimization issues. The research work plan is to study all relevance existing approaches for finding the best solution to fulfil the current requirement. There are many approaches available to solve cloud computing optimization issues. However, there are no methods available to customize data center process execution, resource allocation minimization and quality of service (QoS) constraints with minimal cost in single solutions. The main framework objective is to offer an effective solution to store/search/transmit/ data with minimal cost and time without compromising the QoS constraints. The research study is also planning to find out an effective solution for traffic, data congestion and media streaming issues in a cloud environment. There is list of existing approaches are explained below in details:

\subsection{Performance Optimized Algorithm [15]}

The proposed techniques are deployed with Intel i6 Core processor, with 16 GB RAM, 60 GB Performance optimized algorithm is highly dedicated to optimizing the execution time of data center. Main attention of this methodology minimizes the data process time and response time during data transmission from one data center to another cloud data center. The service broker chooses the cloud data center according to best response time. Load balancing algorithms are designed to optimize the load of the cloud data center and user location taking into consideration transfer delay. However, the techniques do not consider the VM cost and bandwidth optimizations 


\subsection{Round Robin Algorithm (RR) [15]}

Round Robin Algorithm is used for load balancing optimization. The technique divides the time into multiple time slots, and each user base is assigned to a particular time slot for data execution. Round Robin technique initially works by choosing VM randomly and then assigning the request to a virtual machine in a circular queue in the cloud data center. Every assigned VM node shifted to endpoint after allocated request. However, the method does not care the state of VM like whether it's heavily loaded or lightly loaded.

\subsection{Throttled Algorithm [15]}

Throttled Algorithm is designed to optimize the load of VM in cloud computing environment. The technique maintains an index of all VMs with respective state. Once, new request reaches, the table is parsed by load balancer to find out the available VM to allocate the task when VM id returns to cloud data center controller that assigns the request to specific virtual machine. If VM is not available, then it will return -1to data center controller. The alert of new allocation is frequently communicated by data center controller to load balancer after VM allocation/de-allocation. However, the technique is not feasible to minimize the data center execution time.

\subsection{Equally Spread Present Execution Load Algorithm (ESPEL) [15]}

The similarly Spread Present Execution Load methodology is also called as Active Monitoring Load Balancing algorithm. The technique functionality is almost equal to throttled algorithm but change in the VM table indexing. The method maintains all VM table indexing with some allocated request. Once, new request reaches load balances parse VM indexing table to find least loaded VM to allocate the task. Once new request allocation comes then VM count will be increased. Once request is de-allocated VM count will be decreased and alert will be sent to data center controller and users. However, the technique does not care virtual machine cost, data congestion and delay latency.

\subsection{Fuzzy Logic Based Energy-A ware Resource Scheduling Algorithms [1]}

Fuzzy logic based energy-aware resource scheduling framework is designed for both homogenous and heterogeneous cloud. The method focuses on to map the cloud computing workload to enhance resource utilization and the energy utilization with other QoS constraints. The technique also focuses self-optimization like energy, resource allocations and other QoS parameters in cloud environment. However, the technique fails to focus on traffic and data congestion in cloud environment.

\subsection{Adaptive VM Resource Scheduling Algorithm [2]}

Adaptive VM resource scheduling strategy is introduced for both auction technique and numerous aspects like bandwidth utilization and auction time limit. In the method contains two parts namely i) cloud property evaluation \&VM configuration, ii) client auction payment technique. The methods are highly dedicated to sequencing the client's bid on assigned timeline to identify the potential client. The payment of the network resources is negotiated to fix the final price for respective client. The technique does not consider data center execution and request processing time with QoS constraints.

\subsection{Optimization-based Robust Routing Algorithm [3]}

An optimization-based robust routing method (ORRM) developed to offer solution for the steadiness and the energy efficiency is sues in cloud computing environments. To fulfil the QoS demands, the ISP generally utilize the unnecessary design with unpredicted situations of system jamming. Although the method solves QoS issues, energy-efficiency and robustness in cloud environment. But, the method does not care VM optimization and VM cost optimization.

\subsection{DVFS Enabled Efficient-energy Workflow Task Scheduling [4]}

DVFS enabled Efficient-energy Workflow Task Scheduling (DEWTS) is introduced to compute the energy keeps during DVFS method for similar application in the heterogeneous allocated environments. Performance of DEWTS is evaluated through various types' comprehensive experiments. However, the technique is unable to maintain reliable media data transmission without affecting the quality of constraints.

\subsection{Dynamic Fair Priority Optimization Task Scheduling Algorithm [5]}

Dynamic Fair Priority Optimization Task Scheduling methodology worked based on "Weighted Fair Queuing" mechanism to enhance the QoS. The techniques minimize long waiting priority queue and fulfilling QoS constraints at every level. The method is unable to minimize the delay and data congestions is sues. 


\subsection{A dynamic Energy-Efficient Migration and Consolidation Algorithm [6]}

A dynamic energy-efficient migration and consolidation algorithm are developed for replacing the CPU twice threshold methodology to trigger the migration of virtual machines. In the technique improves energy efficiency in data center. It minimizes the amount of virtual machines migration and energetic physical hubs efficiently. However, method is unable to consider dat center executions issues and physical resource minimization.

\subsection{Combinatorial Double Auction Resource Allocation (CDARA) Model [10]}

A combinatorial double auction mechanism is introduced for asset allotment in cloud computing environment. It explains a one-sided combinatorial auction by numerous clients' behalf of single cloud service supplier. CDARA effectively allocate the assets in a cloud computing depends on a combinatorial double auction mechanism. However, the method fails to optimize the load \& bandwidth issues.

\subsection{Green Scheduling Algorithm [18]}

Green Scheduling method is combined by means of a neural network forecaster for optimizing cloud server power expenditure. The predictor contains high accuracy with less overhead to suitable in real active time. The utilization of predictor is to support the method smartly to create suitable turning off/on results. The method evaluates needed active workload on the cloud servers. The method reduces the total cost of owners hip to as sure the QoS. However, the technique does not focus on VM cost and bandwidth optimization is sues.

\subsection{Multi-Objective Genetic Algorithm [19]}

Multi-Objective Genetic Mechanism (MO-GM) is developed to solve energy consumption issues. The technique offers dynamic selection mechanism for real-time requirement according to user. However, some services may be CPU intensive whereas others are I/O-intensive. Cloud resources are required to be allocated not only to fulfil the Quality of Service (QoS) requirements. The technique does not care data center execution issues and VM optimization issues.

\subsection{Deadline Aware Two Stage Scheduling Algorithm [20]}

Deadline Aware Two Stage Scheduling algorithm is designed to complete the user-specific task with minimal time. The method schedules the number of job request to allocated necessary cloud services for every job request. The scheduler accepts tasks from numerous clients and allots the VM as assets by scheduling the task requests. However, the technique does not become feasible for cloud optimization issues, bandwidth optimization is sues and data transmissions issues.

\subsection{A Task-oriented Resource Allocation [21]}

A task-oriented resource allocation method is designed by using mutual pair wise evaluation matrix and the Analytic Hierarchy Process (AHP).The technique is allotted to the jobs along with the mass of every job. Since, loads and the position of jobs will be untrustworthy if discrepancy exists; a lot of learning's have been focused on the inconsistency issue in decision making. But, the majority of the previous methodologies are not precise sufficient to enhance the reliability ratio. Table 1 is illustrated in comparative study of existing approaches. 
Table 1. Comparative Study of Existing Approaches

\begin{tabular}{|c|c|c|}
\hline Technique & Merits & Demerits \\
\hline $\begin{array}{l}\text { Performance Optimized } \\
\text { Algorithm }\end{array}$ & $\begin{array}{l}\text { It optimizes the data center processing time. } \\
\text { The technique time and processing time } \\
\text { during data transmission from once data } \\
\text { center to another data center }\end{array}$ & $\begin{array}{l}\text { The techniques do not consider the VM cost } \\
\text { and bandwidth optimizations }\end{array}$ \\
\hline Round Robin Algorithm(RR) & $\begin{array}{l}\text { The technique divides the time into multiple } \\
\text { time slots, and eachuser base is as signed to a } \\
\text { particular time slot for data execution }\end{array}$ & $\begin{array}{l}\text { The method does notcare the state of VM } \\
\text { like whether it's heavily loaded or lightly } \\
\text { loaded. }\end{array}$ \\
\hline Throttled Algorithm & $\begin{array}{l}\text { It optimizes the load of VM in cloud } \\
\text { computing environment. } \\
\text { The technique manages a directory of all } \\
\text { virtual machines with respective condition. }\end{array}$ & $\begin{array}{l}\text { The technique is not feasible to minimize the } \\
\text { data center execution time }\end{array}$ \\
\hline $\begin{array}{l}\text { Equally Spread Current } \\
\text { Execution Load Methodology } \\
\text { (ESCE) }\end{array}$ & $\begin{array}{l}\text { The technique functionality is almost equal to } \\
\text { throttled algorithm but change in the VM } \\
\text { table indexing. } \\
\text { The methodmaintains all VM table indexing } \\
\text { with some allocated request. }\end{array}$ & $\begin{array}{l}\text { The technique does not care virtual machine } \\
\text { cost, data congestion and delay latency }\end{array}$ \\
\hline $\begin{array}{l}\text { Fuzzy Logic Based Energy- } \\
\text { Aware Resource Scheduling }\end{array}$ & $\begin{array}{l}\text { The method focuses to record the cloud } \\
\text { computing workload to enhance resource } \\
\text { utilization and the energy utilization with } \\
\text { other QoS constraints. } \\
\text { The technique also focuses self-optimization } \\
\text { like energy, resource allocations and other } \\
\text { QoS parameters }\end{array}$ & $\begin{array}{l}\text { The technique fails to focus on traffic and } \\
\text { data congestion in cloud environment. }\end{array}$ \\
\hline $\begin{array}{l}\text { Adaptive VM Resource } \\
\text { Scheduling Mechanism }\end{array}$ & $\begin{array}{l}\text { It introduces the auction technique and } \\
\text { numerous aspects like bandwidth utilization } \\
\text { and auction time limit. } \\
\text { It negotiates to fix the final price for } \\
\text { respective client }\end{array}$ & $\begin{array}{l}\text { The technique does not consider data center } \\
\text { execution and request processing time with } \\
\text { QoS constraints }\end{array}$ \\
\hline Green Scheduling Algorithm & $\begin{array}{l}\text { The technique optimizes cloud server power } \\
\text { consumption. } \\
\text { The predictor contains high accuracy with } \\
\text { less overhead to suitable in real active time }\end{array}$ & $\begin{array}{l}\text { The technique does not focus on VM cost and } \\
\text { bandwidth optimization is sues. }\end{array}$ \\
\hline $\begin{array}{l}\text { Combinatorial Double } \\
\text { Auction Resource Allocation } \\
\text { (CDARA) Method }\end{array}$ & $\begin{array}{l}\text { It introduces for as set distribution in cloud } \\
\text { computing environment. } \\
\text { It explains a one-sided combinatorial auction } \\
\text { with numerous clients' behalf of single cloud } \\
\text { service supplier. }\end{array}$ & $\begin{array}{l}\text { The method fails to optimize the load \& } \\
\text { bandwidth is sues. }\end{array}$ \\
\hline $\begin{array}{l}\text { Deadline Aware Two Stage } \\
\text { Scheduling Strategy }\end{array}$ & $\begin{array}{l}\text { The method schectules number of job request } \\
\text { to allocated necessary cloud services for } \\
\text { every job request. } \\
\text { The scheduler accepts tasks from a variety of } \\
\text { clients and allots the VM as assets by } \\
\text { scheduling the tasks demands. }\end{array}$ & $\begin{array}{l}\text { The technique does not become feasible for } \\
\text { cloud optimization issues, bandwidth } \\
\text { optimizationis sues and data transmissions } \\
\text { is sues. }\end{array}$ \\
\hline $\begin{array}{l}\text { A Task-oriented Resource } \\
\text { Allocation }\end{array}$ & $\begin{array}{l}\text { It assigns the jobs along with the load of } \\
\text { every job. } \\
\text { The technique used mutual pair wise } \\
\text { evaluationmatrix and the Analytic Hierarchy } \\
\text { Process (AHP). }\end{array}$ & $\begin{array}{l}\text { The method does not have enough accuracy } \\
\text { to get better reliability ratio. }\end{array}$ \\
\hline
\end{tabular}

\section{CONCLUSION}

An empirical study reviews all related technique for performance optimization and load balancing scheduling issues. The study also focuses on traffic issues and data center processing delay in cloud environments. There are many approaches available to solve cloud computing optimization issues. However, there are no methods available to customize data center process execution, resource allocation minimization and quality of service (QoS) constraints with minimal cost in single solutions. The main framework objective is to offer effective solution to store/search/transmit/ data with minimal cost and time without compromising the QoS constraints. The research work plan represents different policies and cloud-specific strategies to enhance the performance of cloud application with minimal cost and time. The method can also reduce the bandwidth issues \& minimizes the data center response delay. The technique minimizes the energy consumption for improving the quality of service. The research study is also planning to find out effective solution for traffic, data congestion and media streaming issues in cloud environment.

In future, the paper can be extended to effective framework to access and store unlimited data with minimal cost and time without affective QoS constraints. The work can also be forwarded to maintain reliable media data streaming in cloud environment.

\section{REFERENCES}

[1] Singh, S., and Chana, I.,"EARTH: Energy-aware autonomic resource scheduling in cloud computing", Journal of Intelligent \& Fuzzy Systems, pp.1581-1600, 2016. 
[2] W, Kong., et al., "Virtual machine resource scheduling algorithm for cloud computing based on auction mechanism", Optik - Int. J. Light Electron Opt, pp. 1-6, 2016.

[3] Jiang, D., Xu, Z., Liu, J., Zhao, W., "An optimization-based robust routing algorithm for energy-efficient networks for cloud computing", Springer Science+Business Media New York, pp.1-10, 2015.

[4] Tang, Z., Qi, L., Cheng, Z., Li, K., Khan Samee, U., Li, K.,”An Energy-Efficient Task Scheduling Algorithm in DVFS-enabled Cloud Environment”, Springer Science+Business Media New York, pp. 1-20, 2015.

[5] Saxena, D., Chauhan, R. K., Kait, R., "Dynamic Fair Priority Optimization Task Scheduling Algorithm in Cloud Computing: Concepts and Implementations", I. J. Computer Network and Information Security, Vol. 2, pp. 41-48, 2016.

[6] Li, H., Zhu, G., Cui, C., Tang, H., Dou, Y., He, C., "Energy-efficient migration and consolidation algorithm of virtual machines in data centers for cloud computing", Springer-Verlag Wien, pp. 1-15, 2015.

[7] Goswami, V., Patra, S. S., Mund, G. B., "Performance Analysis of Cloud with Queue-Dependent Virtual Machines", 1st IEEE Int'l Conf. on Recent Advances in Information Technology, RAIT, pp. 1-6, 2012.

[8] Malhotra, R., Jain, P., "Study and Comparison of Various Cloud Simulators Available in the Cloud Computing", International Journal of Advanced Research in Computer Science and Software Engineering, Vol. 3, No. 9, pp. 347-350, 2013.

[9] Pillai, P.S., and RO, s.,"Resource Allocation in Cloud Computing Using the Uncertainty Principle of Game Theory", IEEE SYSTEMS JOURNAL, pp. 1-12, 2014.

[10] Samimi, P., Teimouriy, Mukhtar, M., "A combinatorial double auction resource allocation model in cloud computing" Information Sciences Vol. 357, pp. 201-216, 2016.

[11] Dinh, H. T., Lee, C., Niyato, D., Wang, P., ”A survey of mobile cloud computing: architecture, applications, and approaches", Wirel. Commun. Mob. Comput, Vol. 13, No.18, pp. 1587-1611, 2013.

[12] Sotiriadis, S., and Bessis, N., "An inter-cloud bridge system for heterogeneous cloud platforms", Future Generation Computer Systems, pp. 1-42, 2015.

[13] Zhang, H., Jiang, H., Li, B., Liu, F., Vasilakos, A. V., \& Liu, J., “A framework for truthful online auctions in cloud computing with heterogeneous user demands", IEEE Transactions on Computers, Vol. 65, No. 3, pp. 805-818, 2016.

[14] Vecchiola, C., Pandey, S., Buyya, R., "High-Performance Cloud Computing: A View of Scientific Applications", Pervassive Systems, Algorithms, and Networks (ISPAN), 10th International Symposium, pp. 1-13, 2009.

[15] Agarwal, A., and Jain, S., "Efficient Optimal Algorithm of Task Scheduling in Cloud Computing Environment", International Journal of Computer Trends and Technology (IJCTT),Vol. 9, No.7, pp. 344-349, 2014.

[16] Moura, J., and Hutchison, D., "Review and Analysis of Networking Challenges in Cloud Computing", Journal of Network and Computer Applications, Vol. 60, pp. 113-129, 2016.

[17] Tao, F., LaiLi, Y., Xu, L., Zhang, L., "FC-PACO-RM: A Parallel Method for Service Composition OptimalSelection in Cloud Manufacturing System”, IEEE Transactions on Industrial Informatics, Vol. 9, No.4, pp. 1-12, 2013.

[18] Duy, T. V. T, Sato, Y., Inoguchi, Y, "Performance Evaluation of a Green Scheduling Algorithm for Energy Savings in Cloud Computing", IEEE, 2015.

[19] Liu, J., Luo, X., Zhang, X., Zhang, F., Li, B., "Job Scheduling Model for Cloud Computing Based on MultiObjective Genetic Algorithm", IJCSI International Journal of Computer Science Issues, Vol. 10, No.3, pp. 134139, 2013.

[20] Raju, K., Indukuri, R., Varma1, S. P., Sundari, M.V.R., Moses, G. J., ’Deadline Aware Two Stage Scheduling Algorithm in Cloud Computing", Indian Journal of Science and Technology, Vol. 9, No. 4, pp. 1-10, 2016.

[21] Ergu, D., Kou, G., Peng, Y., Shi, Y., "The analytic hierarchy process: task scheduling and resource allocation in cloud computing environment", Springer Science+Business Media, pp. 1-14, 2014.

[22] Abdullahi, M., Ngadi, M. A., Abdulhamid, S. M., "Symbiotic Organism Search optimization based task scheduling in cloud computing environment", Future Generation Computer Systems, pp. 1-29, 2015.

[23] Kliazovich, D., Tchernykh, A., Johnathan, E. P., Bouvry, P., Khan, S. U., Zomaya, A Y., "CA-DAG: Modeling Communication-Aware Applications for Scheduling in Cloud Computing" J Grid Computing, Vol. 14, pp. 23-39, 2016.

[24] Xiao, Z., Song, W., Chen, Q., "Dynamic Resource Allocation using Virtual Machines for Cloud Computing Environment", IEEE Transaction on Parallel and Distributed Systems, Vol. 24, No. 6, pp. 1107-1117, 2013.

[25] Rodrigo, N., Calheiros, R.N.., Marco, A. S., Netto, M.A.S., Rose, C.A.F.D., Buyya R., "EMUSIM: An Integrated Emulation and Simulation Environment for Modeling, Evaluation, and Validation of Performance of Cloud Computing Applications", Software - Practice And Experience, pp. 1-18, 2012.

[26] Talasila, S., Vani, H., Sai, K., Mani, D., Krishna Reddy, V., "Load Balancing Techniques for Efficient Traffic Management in Cloud Environment", International Journal of Electrical and Computer Engineering (IJECE), Vol. 6, No. 3, pp. 963-973, 2016.

[27] Nadim Akhtar, N. Srinivasan, "Notice of Retraction Intermittently Connected Cloudlet System to Obtain an Optimal Offloading Policy", Indonesian Journal of Electrical Engineering and Informatics (IJEEI), Vol. 4, No.3, 2016.

[28] Ravi Teja Kanakala, V., Krishna Reddy, V., "Performance Analysis of Load Balancing Techniques in Cloud Computing Environment", TELKOMNIKA Indonesian Journal of Electrical Engineering, Vol. 13, No. 3, pp. 568 573,2015 